\title{
Mapping of paternal-sex-ratio deletion chromosomes localizes multiple regions involved in expression and transmission
}

\author{
BF McAllister ${ }^{1}$, LW Beukeboom ${ }^{2}$ and JH Werren ${ }^{3}$ \\ ${ }^{1}$ Department of Biological Sciences, 138 Biology Building, University of Iowa, Iowa City, IA 52242, USA; ${ }^{2}$ Centre for Ecological and \\ Evolutionary Studies, University of Groningen, The Netherlands; ${ }^{3}$ Department of Biology, University of Rochester, Rochester, NY, USA
}

\begin{abstract}
The paternal-sex-ratio (PSR) chromosome in the parasitic wasp Nasonia vitripennis is a submetacentric supernumerary (B chromosome). Males transmit PSR, but after fertilization it causes the loss of the paternal autosomes. Paternal genome loss caused by PSR results in the conversion of a female (diploid) zygote into a male (haploid) under haplodiploid sex determination. In this study, site-specific markers were developed to assay deletion derivatives of PSR. Both polymerase chain reaction and Southern hybridization were used to detect the presence/absence of 16 single-site markers on a set of 20 functional and nine nonfunctional deletion chromosomes. Based on the pattern of marker loss on the deletion chromosomes, the basic organization of PSR was revealed. Two sets of markers were deleted independently, apparently representing the two arms of the
\end{abstract}

submetacentric chromosome. The presence or absence of specific regions was examined in relation to phenotypic characteristics of the deletion chromosomes; ability to cause paternal genome loss, and stability in mitotic cell divisions. Rather than identifying a single region on PSR as being responsible for PSR function, the results suggest that the retention of one of two chromosomal regions is sufficient for causing paternal genome loss. Furthermore, a region was identified that is tightly correlated with mitotic stability, as measured from chromosomal transmission rates. Functional chromosomes with short-arm deletions had high $(\sim 100 \%)$ transmission rates, whereas functional chromosomes with long-arm deletions had low ( 85\%) transmission rates.

Heredity (2004) 92, 5-13, advance online publication, 24 September 2003; doi:10.1038/sj.hdy.6800355

Keywords: B chromosome; deletion mapping; chromosomal segregation; selfish DNA; Nasonia

\section{Introduction}

Selfish genetic elements enhance their own transmission, but are neutral or detrimental to their host organisms (Werren et al, 1988; Hurst and Werren, 2001). Diverse genetic elements have been classified as 'selfish' or 'parasitic', including driving chromosomes, transposable elements, heritable microbes, and others. Driving chromosomes are particularly interesting examples of selfish genetic elements, because they violate Mendel's rule of alternate segregation by being over-represented in the progeny of carriers (Lyttle, 1991, 1993). Well-studied examples of driving chromosomes include segregation distorter and sex ratio in Drosophila (Wu and Hammer, 1991; Lyttle, 1993; Jaenike, 1996), t-complex in Mus (Silver, 1985, 1993), and supernumerary (B) chromosomes in many organisms (Jones and Rees, 1982; Jones, 1991; Camacho et al, 2000). These systems are important illustrations of how selection blindly increases the frequency of the driving chromosome, although this increase may ultimately reduce mean fitness or cause extinction of the population (Lyttle, 1977). As has been recognized by Hastings (1994), there is great potential in the ability to manipulate selfish genetic elements and use

Correspondence: BF McAllister, Department of Biological Sciences, 138 Biology Building, University of Iowa, Iowa City, IA 52242, USA.

E-mail: bryant-mcallister@uiowa.edu

Received 7 August 2002; accepted 7 July 2003 them to control pest species. Before this achievement can be realized, the genetic mechanisms underlying these systems must be understood.

Paternal-sex-ratio (PSR) is a B chromosome in the parasitic wasp Nasonia vitripennis (reviewed in Werren and Stouthamer, 2002). This chromosome is an extreme example of a selfish genetic element, because its mode of transmission causes the elimination of all other genetic information present in carrier wasps. Only males carry PSR. After fertilization with a PSR-bearing sperm, the paternal autosomes form into a chromatin mass and fail to divide (Werren et al, 1987; Reed and Werren, 1995). The PSR chromosome segregates with the haploid maternal autosomes in apparently normal zygotic development. Exploiting haplodiploid sex determination, PSR converts a female (diploid) zygote into a male (haploid). Each generation, PSR remains in a haploid male with mitotic spermatogenesis, and thus it avoids meiotic segregation entirely. So far, the only phenotypic trait associated with PSR is that females mated to PSR-carrying males produce all-male progenies (Werren et al, 1981; Beukeboom, 1994). As only sperm transmit PSR, the proportion of male progeny carrying PSR is directly dependent on the fertilization rate (Werren and van den Assem, 1986; Beukeboom and Werren, 1993a).

Although PSR causes paternal genome loss following fertilization, the timing of PSR's action and the molecular mechanism of paternal genome loss remain unknown. PSR either functions during spermatogenesis or within 
the fertilized egg prior to the first division of the zygote. At either stage, PSR must be immune to its own action. Two primary models have been proposed for the mechanism of paternal genome loss caused by PSR (Werren, 1991; Beukeboom and Werren, 1993b). First, the 'genic model' postulates that a product encoded by PSR imprints the paternal autosomes during spermatogenesis. This imprint interferes with the normal processing of the paternal autosomes after fertilization. Alternatively, the 'sink model' postulates that a repetitive sequence on PSR binds a product needed by the paternal autosomes. This titration of a product away from the paternal autosomes could occur either during spermatogenesis or following fertilization in the egg.

In a previous examination of the mechanism underlying paternal genome loss caused by PSR, a deletion analysis was performed (Beukeboom and Werren, 1993b). Deletion derivatives of PSR were generated by two methods: (i) irradiating adult males and (ii) transmitting PSR through Wolbachia-induced incompatible crosses between $N$. vitripennis strains. The deletion chromosomes were classified into two groups based on their ability to cause paternal genome loss, functional and nonfunctional. Functional deletion chromosomes retained the ability to cause paternal genome loss, and these were transmitted only to male offspring (Beukeboom and Werren, 1993b). For some of the functional deletion chromosomes, however, a substantial number of female progeny were produced from carrier males. Examination of two such chromosomes indicated that the mitotic instability causes some males to exhibit somatic and testicular mosaicism (Beukeboom et al, 1992). The latter results in some sperm not receiving the chromosome, and therefore females develop following fertilization with these non-PSR sperm. Nonfunctional deletion chromosomes were also generated, and were incapable of causing paternal genome loss. These nonfunctional chromosomes were transmitted from carrier males to fertilized eggs, where normal diploid (female) development occurs. The nonfunctionals were also transmitted by females, but to only 5-10\% of their eggs (Beukeboom and Werren, 1993b). Low transmission rates in females are probably due to improper segregation and loss of the PSR univalent during meiosis.

In addition to classifying the deletion chromosomes by type, the chromosomes were assayed for their content of tandemly repeated sequences (Beukeboom and Werren, 1993b). Five distinct families of tandemly repetitive DNA can be distinguished on PSR (Eickbush et al, 1992). One problem with using these repetitive sequences to assay for the presence of specific chromosomal regions, however, is that each repeated sequence might be localized in multiple arrays. Site-specific markers would provide a definite improvement for detecting the presence/absence of specific regions on deletion chromosomes. In this study, two methods were developed to detect site-specific markers on the PSR deletion chromosomes. Assays using the polymerase chain reaction (PCR) have been developed to detect the presence of five sequence-tagged sites (STSs) on PSR. Assays were also developed to determine the presence/absence of anonymous restriction fragments on the deletion chromosomes. Profiles of these markers on a set of deletion chromosomes are compared to functionality and chromosomal stability.

\section{Materials and methods}

\section{Strains}

The strains of $N$. vitripennis used in this study were cultured at $25^{\circ} \mathrm{C}$ on flesh fly (Sarcophaga bulata) pupae. All except one of the deletion strains were created and reported by Beukeboom and Werren (1993b). The one exceptional strain was EMS7, a nonfunctional PSR chromosome derived by feeding an adult male PSR carrier with sugar water containing 0.05 M EMS (methanesulfonic acid methyl ester; Perrot-Minnot and Werren, 2001). Since their creation, the deletion lines have been maintained as diapause larvae where they were stored at $4^{\circ}$ and cycled through the adult stage (1-3 generations) approximately once a year using the high-fertilizing MI strain of $N$. vitripennis. Some of the strains have been lost from culture, and in these cases DNA was extracted from previously frozen adult wasps.

Wild-type and PSR carriers do not differ phenotypically. Therefore, a genetic screen is necessary to ensure the presence of the chromosome. The PSR chromosome contains several unique tandemly repeated sequences that are absent on the autosomes (Eickbush et al, 1992), and dot-blot assays (Beukeboom and Werren, 1993b) based on the hybridization to these repeats were used during standard maintenance and transmission assays (described below) for a postinsemination determination of PSR presence in adult wasps. For the functional deletion chromosomes, single male wasps that potentially carried PSR were mated with virgin MI females. The males were screened for the presence of PSR using the dot-blot procedure while the females were provided hosts for laying eggs. Male progeny from all-male or high-male families with a PSR-carrying father were collected for DNA extraction. Nonfunctional PSR chromosomes were transmitted from a carrier male to fertilized eggs that develop into females. For these lines, males potentially carrying PSR were mated to MI females and subsequently screened using the dot-blot procedure. Females with a father that carried PSR were collected for DNA extraction. DNA was extracted from adult wasps pooled from multiple families within each chromosomal line. Standard techniques were followed for phenol/chloroform extraction and ethanol precipitation to purify the DNA (McAllister, 1995).

\section{PCR assays}

In a previous study (McAllister, 1995), five unique regions of the PSR chromosome were isolated in $\lambda$-clones. The shared feature among these regions is the presence of the retrotransposable element NATE, although each region has unique sequences flanking the element. By exploiting unique features in each of the five regions (Figure 1), PCR assays were developed to detect their presence. Each of these regions is essentially an STS on the PSR chromosome. Primer sequences, annealing temperatures (ATs) for the reactions, and size of the amplified products for the five assays are given in Table 1. A positive control reaction was used to verify the presence of PSR and verify that each DNA sample was PCR competent. This reaction used two primers flanking the RT region of NATE (Table 1). These primers amplify elements on PSR, but will not amplify elements in the autosomal DNA of $N$. vitripennis. 


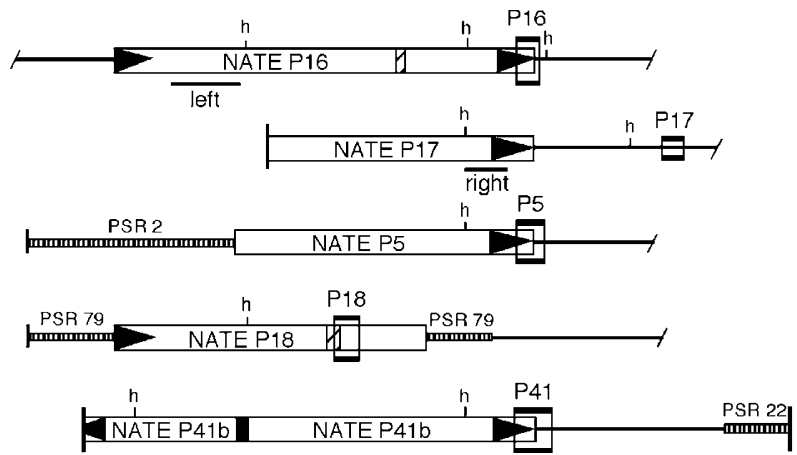

$-1 \mathrm{~kb}$

Figure 1 Five cloned regions of the PSR chromosome containing site-specific markers. Boxes in each of the regions represent the area amplified in each of the PCR assays. Regions used as the left and right probes in the Southern assays are identified, and mapped restriction sites for HindIII (h) that generate hybridizing fragments are indicated. Locations of the PSR2, PSR79, and PSR2 tandemly repeated sequences are also indicated.

Table 1 Primer sequences, annealing temperatures, and sizes of amplified products for the PCR assays

\begin{tabular}{llcc}
\hline Marker & Primer sequences & $\begin{array}{c}\text { Ann. } \\
\text { temp. } \\
(\mathrm{deg})\end{array}$ & $\begin{array}{c}\text { Size } \\
(\mathrm{bp})\end{array}$ \\
\hline P5 & $\begin{array}{l}\text { N8: ACGATTACATAAAGCATAG } \\
\text { P5-1: CATGCTTAGAAAGTATGGC }\end{array}$ & 50 & 700 \\
P16 & $\begin{array}{l}\text { N7: CACGAGGCATCATCTGCG } \\
\text { P16-1: CGAACTTGAACGATATCATCT }\end{array}$ & 60 & 600 \\
P17 & $\begin{array}{l}\text { P17-1: TGGAATTCAGACCAAGATCG } \\
\text { P17-3: CAGAATCCTATAGCAGTTCG }\end{array}$ & 56 & 1500 \\
P18 & $\begin{array}{l}\text { N13: CAATGGGTTTGACTCGTTCG } \\
\text { P18-1: GATGTAGACTTGAGATCGTG }\end{array}$ & 58 & 600 \\
P41 & $\begin{array}{l}\text { N8: ACGATTACATAAAGCATAG } \\
\text { P41-1: ACATCTACGCACAAGCAG }\end{array}$ & 54 & 600 \\
Control & $\begin{array}{l}\text { N12: GCCTCCCTCTCGTCTGCA } \\
\text { N13: CAATGGGTTGACTCGTTCG }\end{array}$ & & \\
& & & \\
\end{tabular}

The PCR assays were conducted in $25 \mu \mathrm{l}$ reactions with $1 \times$ buffer, $2.5 \mathrm{mM} \mathrm{MgCl}_{2}, 200 \mathrm{nM}$ of each primer, $100 \mu \mathrm{M}$ of each dNTP, and $2 \mathrm{U}$ of Taq polymerase. Solutions of DNA from the mass extractions were diluted to an approximate concentration of $25 \mathrm{ng} / \mu \mathrm{l}$ in TE and $1 \mu \mathrm{l}$ was used as template for each reaction. Controls of genomic DNA from PSR(MI) and MI, a $\lambda$-clone containing the STS, and no DNA were always assayed in parallel to the deletion lines. ATs varied for the different assays as listed in Table 1 . The standard reaction was: $2 \times\left(95^{\circ}, 2 \mathrm{~min}\right.$; AT, $\left.1 \mathrm{~min} ; 72^{\circ}, 2 \mathrm{~min}\right)$ followed by $35 \times\left(95^{\circ}, 0.5 \mathrm{~min}\right.$; AT, $\left.1 \mathrm{~min} ; 72^{\circ}, 1 \mathrm{~min}\right)$. Owing to a longer product for P17, extension times at $72^{\circ}$ were 3 and $1.5 \mathrm{~min}$, respectively. About $5 \mu \mathrm{l}$ samples of the reaction products were assayed on $1.0 \%$ agarose gels.

\section{Restriction fragment assays}

Two assays were developed for screening the deletion chromosomes for the presence of specific restriction fragments. Each assay involves detecting restriction fragments containing insertion sites at either end of the retrotransposon NATE. Two plasmid clones were obtained by subcloning from $\lambda$-clones containing copies of NATE (Figure 1). The clones have inserts representing regions near the two ends of the element, one referred to as the left (p16EH27) and the other right (p17EH5). Insertion sites were assayed by digesting genomic DNA with HindIII and visualizing restriction fragments through hybridization of the probes. Restriction sites for HindIII were located internally within NATE relative to the location of each probe, so the size of each hybridizing fragment was determined by the position of the nearest HindIII site in the flanking sequence of each element.

Total genomic DNA from the deletion lines, wild-type PSR, and the MI strain without PSR was restriction digested with HindIII. Each time deletion lines were screened, the wild-type PSR and MI were included on the gel. DNA was electrophoresed through $0.8 \%$ agarose gels and transferred to nylon membranes. Probes were prepared by initially amplifying the inserts from plasmid (Bluescript) DNA using the T3 and T7 primers, which flank the inserts. Approximately $25 \mathrm{ng}$ of the PCR product was labeled with $\left[\alpha^{32} \mathrm{P}\right] \mathrm{dATP}$ using random primer labeling (BRL). Probes were hybridized to the membranes at $65^{\circ}$ for $16 \mathrm{~h}$ in a solution of $5 \times \mathrm{SSC}$, $5 \times$ Denhardt's solution, $125 \mu \mathrm{g} / \mathrm{ml}$ ctDNA, and $1 \%$ SDS. The membranes were rinsed at increasing stringency until a final rinse in $0.2 \times$ SSC and $0.1 \%$ SDS at $65^{\circ}$. Membranes were exposed to film at $-80^{\circ}$ with an amplifying screen. The same membranes were hybridized independently to multiple probes. Boiling $0.1 \%$ SDS was poured over the membranes to remove probes between hybridizations.

\section{Transmission assay}

Deletion strains were assayed to determine chromosomal transmission rates from male wasps. Potential carriers were pair-mated to virgin females (MI); each female was provided two hosts for laying eggs, and each male was screened by dot-blot analysis to determine if it carried the deleted PSR chromosome. Transmission rates of deletion chromosomes were estimated using two methods, phenotypic and genotypic assays. Estimates for functional chromosomes are based on the fertilization rate of the females, so control crosses to estimate the fertilization rate were conducted simultaneously using virgin females in pair-matings with MI males (non-PSR carriers). These females were also given two hosts.

In the phenotypic assays, the numbers of males (M) and females $(\mathrm{F})$ were counted in the progeny $(N=\mathrm{M}+\mathrm{F})$ from male carriers. Using the mean fertilization rate $(x)$ from the control cross, the transmission rate of the chromosome was estimated using the following equation:

$$
t_{\mathrm{p}}=\frac{\mathrm{M}-\mathrm{N}(1-x)}{x N}
$$

This transmission assay is based on the excess number of male progeny, assuming a standard proportion were produced from unfertilized eggs, to estimate the percentage of male progeny that developed from fertilized eggs following the loss of the paternal genome. The rate maximizes at $100 \%$ when only males are obtained. For each deletion chromosome, phenotypic transmission 
rates were estimated for multiple families for which descriptive statistics were calculated.

Genotypic assays of transmission rates for some functional deletion chromosomes were also performed. For this assay, 20 male progeny from each of 15-20 families representing each functional deletion chromosome were screened by dot-blot analysis. This measures the proportion $(p)$ of male progeny that received the chromosome. In each of the families, the transmission rate of the chromosome was estimated as follows:

$$
t_{\mathrm{g}}=\frac{p \mathrm{M}}{x \mathrm{~N}}
$$

Based on the frequency of PSR in the adult male progeny, $t_{\mathrm{g}}$ estimates the transmission rate assuming a standard fertilization rate. A subsample of families was used to estimate $t_{\mathrm{g}}$ for each chromosome, and only families with at least 20 males were assayed. If the proportion of male progeny with PSR $(p)$ exceeded the estimated fertilization rate $(x)$, the estimate of $t_{\mathrm{g}}$ was greater than $100 \%$, and in these families $t_{\mathrm{g}}$ was set at $100 \%$.

Measurement of transmission rates of nonfunctional chromosomes requires a genotypic assay, because there are no phenotypic effects. Transmission rates from males carrying the chromosomes were determined by dotblotting their female progeny. The percentage of these progeny that carried the chromosome was used as a direct estimate of transmission rate.

\section{Results}

\section{Presence of markers on deletion chromosomes}

In total, 20 functional and nine nonfunctional deletion chromosomes were screened using the five PCR assays, which detect the presence of STS markers on the PSR chromosome. Table 2 lists the presence/absence of the five STS markers for each deletion chromosome. Four markers (P5, P41, P16, and P17) were almost completely associated within these lines $(P<0.01$, pairwise exact tests). All four markers were present on 12 chromosomes, whereas 14 chromosomes had all four markers deleted. Only three exceptions were observed in that P5, $\mathrm{P} 41$, and P16 were each present on a single chromosome when the others were deleted. As is apparent in Table 2, the P18 marker showed a pattern of deletion independent of the other four markers $\left(P>0.05\right.$, pairwise $\chi^{2}$ tests).

A clear difference was observed for the frequency of marker loss between functional and nonfunctional chromosomes (Table 2). Seven $(35 \%)$ of the functional chromosomes did not have any markers deleted. Four (20\%) functional chromosomes lost P18 only, nine (45\%) lost at least three of P5, P41, P16, and P17, and no markers were detected on one $(5 \%)$ chromosome. However, seven $(78 \%)$ nonfunctional chromosomes lost all of the markers, and only two (22\%) had any markers present. A significant difference is observed between functional and nonfunctional chromosomes missing P18 or at least one of P5, P41, P16, and P17 versus chromosomes missing P18 and at least one of P5, P41, P16, and P17 $(P<0.01$, exact test).

Assays for the presence of restriction fragments on the PSR deletion chromosomes were conducted on the lines where the quality and quantity of DNA were permissive.
Table 2 Presence or absence of five STS markers on PSR deletion chromosomes

\begin{tabular}{|c|c|c|c|c|c|}
\hline & \multicolumn{5}{|c|}{ Markers } \\
\hline & P18 & P5 & $P 41$ & P16 & P17 \\
\hline \multicolumn{6}{|c|}{ Functional } \\
\hline E105 & + & + & + & + & + \\
\hline E203 & + & + & + & + & + \\
\hline E228 & + & + & + & + & + \\
\hline H003 & + & + & + & + & + \\
\hline H007 & + & + & + & + & + \\
\hline M021 & + & + & + & + & + \\
\hline N033 & + & + & + & + & + \\
\hline E146 & - & + & + & + & + \\
\hline E163 & - & + & + & + & + \\
\hline E230 & - & + & + & + & + \\
\hline F491 & - & + & + & + & + \\
\hline E242 & + & + & - & - & - \\
\hline E036 & + & - & + & - & - \\
\hline E087 & + & - & - & - & - \\
\hline E121 & + & - & - & - & - \\
\hline E128 & + & - & - & - & - \\
\hline I001 & + & - & - & - & - \\
\hline M006 & + & - & - & - & - \\
\hline M008 & + & - & - & - & - \\
\hline E306 & - & - & - & - & - \\
\hline \multicolumn{6}{|c|}{ Nonfunctional } \\
\hline E133 & - & + & + & + & + \\
\hline M001 & - & - & - & + & - \\
\hline H019 & - & - & - & - & - \\
\hline L002 & - & - & - & - & - \\
\hline N009 & - & - & - & - & - \\
\hline N013 & - & - & - & - & - \\
\hline N029 & - & - & - & - & - \\
\hline EMS7 & - & - & - & - & - \\
\hline F599 & - & - & - & - & - \\
\hline
\end{tabular}

The assays were performed on 18 functional and six nonfunctional deletion chromosomes. For the assay using the left end of NATE as a probe, four major hybridizing bands were detected and scored. The approximate size of the scored bands and the profiles of the chromosomes are presented in Table 3. An additional strongly hybridizing band $(8.7 \mathrm{~kb})$ was visualized, but the intensity of the band indicated that it was either a doublet or a triplet. Owing to potential ambiguity, it was not scored. When the right end of NATE was used as a probe, seven hybridizing bands were scored and the profiles of the chromosomes are presented in Table 3. Again, the intensity of one hybridizing band $(16 \mathrm{~kb})$ was suggestive of a doublet, so it was not scored.

Following both the PCR and restriction fragment assays, seven functional chromosomes did not exhibit any apparent deletions. Deletions of at least one marker were detected for 13 functional and nine nonfunctional chromosomes. Figure 2 represents the compiled data for the PCR and restriction fragment markers based on their inferred positions on the PSR chromosome. Nomenclature for the restriction fragments is given as NL or NR, referring to markers detected with either the left or right end of NATE, followed by the approximate size (in $\mathrm{kb}$ ) of the hybridizing band.

Data on the composition of repetitive sequences for the deletion chromosomes were obtained from previous studies that employed dot-blot hybridization 
Table 3 Presence or absence of restriction fragments on PSR deletion chromosomes

\begin{tabular}{|c|c|c|c|c|c|c|c|c|c|c|c|}
\hline & & & & & & & & $T E r$ & & & \\
\hline & $4.7^{a}$ & 5.1 & 6.2 & 13 & 1.3 & 2.2 & 3.2 & 3.7 & 4.2 & 6.0 & 8.8 \\
\hline Functio & & & & & & & & & & & \\
\hline E203 & + & + & + & + & + & + & + & + & + & + & + \\
\hline E228 & + & + & + & + & + & + & + & + & + & + & + \\
\hline H003 & + & + & + & + & + & + & + & + & + & + & + \\
\hline H007 & + & + & + & + & + & + & + & + & + & + & + \\
\hline M021 & + & + & + & + & + & + & + & + & + & + & + \\
\hline N033 & + & + & + & + & + & + & + & + & + & + & + \\
\hline E230 & NA & NA & NA & NA & + & + & + & + & + & + & + \\
\hline E163 & + & - & + & + & + & + & + & + & + & + & + \\
\hline F491 & + & - & + & + & + & + & + & + & + & + & + \\
\hline E146 & + & - & - & + & - & + & + & + & + & + & + \\
\hline E306 & - & + & + & - & + & - & + & - & - & - & + \\
\hline E087 & - & + & + & - & + & - & + & - & - & - & + \\
\hline E121 & - & + & + & - & + & - & + & - & - & - & + \\
\hline E128 & - & + & + & - & + & - & + & - & - & - & + \\
\hline M006 & - & + & + & - & + & - & + & - & - & - & + \\
\hline M008 & - & + & + & - & + & - & + & - & - & - & + \\
\hline E036 & + & + & + & - & + & - & - & + & - & + & + \\
\hline E242 & - & + & + & - & + & - & - & - & - & - & + \\
\hline Nonfun & & & & & & & & & & & \\
\hline E133 & + & - & + & + & + & + & + & + & + & + & + \\
\hline N009 & - & - & + & - & + & - & + & - & - & - & + \\
\hline N013 & NA & NA & NA & NA & + & - & + & - & - & - & + \\
\hline N029 & - & - & + & - & + & - & + & - & - & - & + \\
\hline EMS7 & - & - & + & - & + & - & + & - & - & - & + \\
\hline F599 & - & - & - & - & - & - & + & - & - & - & + \\
\hline
\end{tabular}

$\mathrm{NA}=$ not assayed

a Size of the hybridizing fragment.
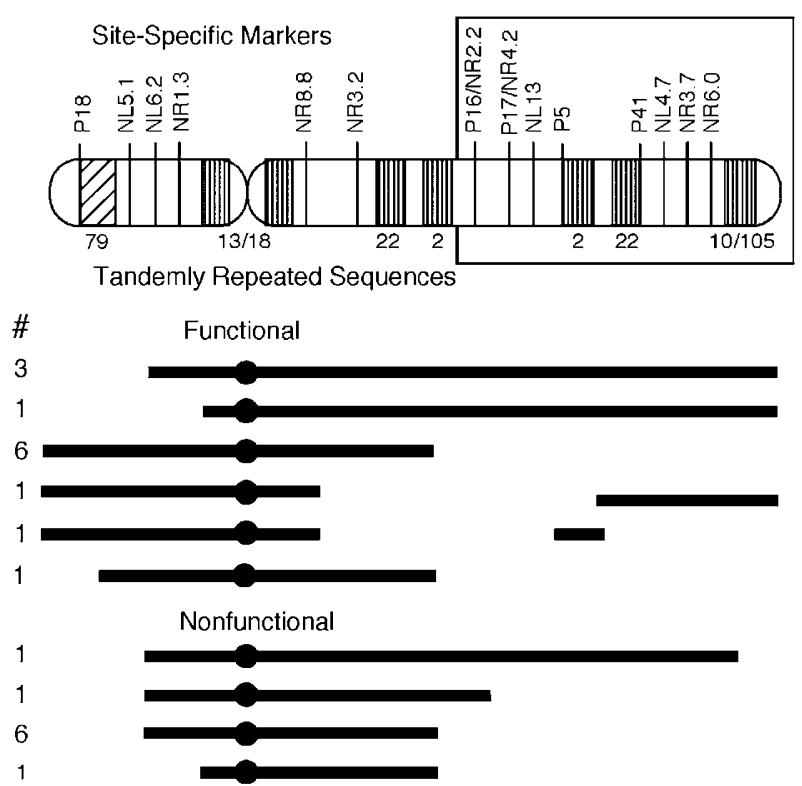

Figure 2 Composite map of the PSR chromosome. Deletion chromosomes are organized in groups having the same profiles with black lines representing retained regions on the chromosome. In placing markers on the chromosome, it is assumed that the long arm contains the larger number of markers and that terminal deletions are more frequent than interstitial deletions. Order of markers in the boxed region is unresolved.

(Beukeboom and Werren, 1993b). The Southern blots of HindIII-digested DNA from some deletion chromosomes were also screened with the PSR79 and PSR2 repeats, thus providing a comparison with the dot-blot results.
Positions of the tandemly repeated sequences are presented for the PSR79, PSR2, PSR22, PSR10/105, and PSR13/18 families in Figure 2.

The organization of the five cloned regions of PSR provides a basis for comparing among the PCR, restriction fragment, and repetitive DNA composition of the deletion chromosomes. For two of the cloned regions detected by PCR assays, the sizes of HindIII restriction fragments at the ends of the elements are known from restriction maps of the clones (Figure 1). These elements provide independent verification between the PCR and restriction site assays, and complete congruence was observed (Tables 2 and 3, Figure 2).

Although the other markers do not overlap in the cloned regions, blocks of codeleted markers were revealed. The markers fall into two independently deleted regions, and these are interpreted as representing the two arms of the chromosome (Figure 2). Cytological investigation indicates that PSR is a submetacentric chromosome (Nur et al, 1988; Reed, 1993), and markers from both chromosomal arms were apparently assayed. Four markers are assumed to be located on the short arm of the submetacentric, whereas 11 are assumed to be located on the long arm. However, this has not been confirmed cytologically. The NR8.8 restriction fragment was not lost on any of the chromosomes assayed. Therefore, its location is unknown except we infer that it is tightly associated with the centromere.

The majority of chromosomes with deletions of the presumed long arm exhibit loss of a block of 10 markers. Only single chromosomes were characterized where all 10 markers were not lost. Owing to the small sample, these markers in the long arm of the chromosome cannot 
be confidently organized in a linear fashion on the chromosome. Furthermore, interstitial deletions apparently occurred in at least two cases (Figure 2).

\section{Transmission rates}

Functional chromosomes with detectable deletions and nonfunctional chromosomes were tested for their rates of transmission from male wasps that were dot-blot positive for the chromosomes. One estimate of functional PSR transmission rate is based on a phenotypic assay by measuring the sex ratio of the progeny from carrier males relative to control males. Measurements of phenotypic transmission rates were originally obtained following the creation of the deletion lines, and were subsequently obtained for a subset of chromosomes 5 years after establishment of the lines. Estimated transmission rates of functional deletion chromosomes are presented in Table 4 . These estimates can be highly skewed, due to most families exhibiting high transmission rates and a few with very low rates; therefore, median values will be used in this presentation.

One interesting observation is that the transmission rates are correlated with the profiles of the functional deletion chromosomes $\left(r^{2}=0.86\right)$. Chromosomes with apparent terminal short-arm deletions exhibited high transmission, with phenotypic transmission rates of $100 \%$ originally and in the later analysis. In contrast, chromosomes with apparent terminal long-arm deletions exhibited low transmission (64-97\%) in the original assay, without improvement in the subsequent assay (63-93\%). E036 exhibits a marker profile that is consistent with having an interstitial deletion of the long arm, and it also exhibits a high $(100 \%)$ transmission rate.

Another estimate of transmission rate was obtained for the established chromosomes using a genotypic assay that detects the presence of the chromosome in adult male offspring of a carrier male. The expectation is for unity of the phenotypic and genotypic measurements, but the phenotypic rate will be higher if the chromosome causes paternal genome loss, but is subsequently lost. The estimated genotypic transmission rate was generally less than the phenotypic rate (Table 5), which indicates that some loss of the deletion chromosome is occurring during development of male wasps that result from PSR

Table 4 Phenotypic transmission rates for functional deletion chromosomes upon formation and after establishment in culture

\begin{tabular}{lcc}
\hline Line & Original & Established \\
\hline F491 $^{\mathrm{s}}$ & $1.00,0.98,0.00,18$ & $1.00,1.00,0.00,16$ \\
E163 $^{\mathrm{s}}$ & $1.00,0.95,0.05,19$ & $1.00,0.99,0.00,15$ \\
E146 $^{\mathrm{s}}$ & $1.00,0.85,0.13,18$ & $1.00,0.97,0.02,43$ \\
E230 $^{\mathrm{s}}$ & $1.00,1.00,0.00,19$ & \\
E306 $^{1}$ & $0.97,0.81,0.08,18$ & $0.63,0.64,0.09,29$ \\
E242 $^{1}$ & $0.82,0.76,0.05,18$ & $0.81,0.76,0.06,27$ \\
M008 $^{1}$ & $0.98,0.68,0.19,18$ & $0.93,0.77,0.10,30$ \\
I001 $^{1}$ & $0.64,0.53,0.14,4$ & $0.86,0.67,0.14,21$ \\
E128 $^{1}$ & $0.94,0.83,0.07,15$ & \\
M006 $^{1}$ & $0.84,0.77,0.05,18$ & \\
E121 $^{1}$ & $0.73,0.64,0.11,16$ & \\
E087 $^{1}$ & $0.65,0.56,0.17,17$ & \\
E036 $^{\mathrm{i}}$ & $1.00,0.92,0.02,20$ & \\
\hline
\end{tabular}

Chromosomal deletions are identified as sshort arm, llong arm, or ${ }^{i}$ interstitial based on marker results. Transmission rates are reported as median, mean, and variance among families, and the number of families assayed.
Table 5 Comparison of phenotypic and genotypic transmission rates for functional deletion chromosomes

\begin{tabular}{lcccc}
\hline Line & $\mathrm{N}$ & Phenotypic & Genotypic & $\begin{array}{c}\text { Genotypic/ } \\
\text { phenotypic }\end{array}$ \\
\hline F491 $^{\text {s }}$ & 16 & $1.00,1.00,0.00$ & $1.00,0.96,0.07$ & 1.00 \\
E163 $^{\text {s }}$ & 15 & $1.00,0.99,0.03$ & $0.96,0.86,0.24$ & 0.96 \\
E146 $^{\text {s }}$ & 19 & $1.00,1.00,0.00$ & $1.00,0.93,0.15$ & 1.00 \\
M008 $^{1}$ & 20 & $0.93,0.86,0.18$ & $0.84,0.79,0.24$ & $0.90^{*}$ \\
I001 $^{1}$ & 15 & $0.92,0.80,0.21$ & $0.74,0.71,0.18$ & $0.81^{*}$ \\
E306 $^{1}$ & 20 & $0.66,0.70,0.23$ & $0.59,0.61,0.18$ & $0.89^{*}$ \\
E242 $^{1}$ & 20 & $0.80,0.77,0.20$ & $0.76,0.74,0.18$ & 0.95 \\
\hline
\end{tabular}

$N$ represents the number of families assayed, and transmission rates are reported as median, mean, and variance among families.

Chromosomal deletions are identified as short arm and long arm. ${ }^{*}$ Significance $(P<0.05)$ with the Wilcoxon signed-rank test.

Table 6 Transmission rates through males for nonfunctional deletion chromosomes upon formation and after establishment in culture

\begin{tabular}{lll}
\hline Line & Original & Established \\
\hline F599 & $0.59,333$ & $0.99,320$ \\
H019 & $0.94,18$ & $0.96,74$ \\
E133 & $1.00,18$ & \\
H019 & $0.94,18$ & \\
N013 & $0.40,10$ & \\
L002 & $0.75,992$ & \\
N009 & $0.50,10$ & \\
N029 & $0.80,10$ & \\
EMS7 & $0.95,39$ & \\
\hline
\end{tabular}

Transmission rates reported as rate and number of progeny tested.

action (elimination of the paternal genome). Loss may be a result of random mitotic instability, or possibly due to the functional chromosome being inefficiently protected from its own action and thus eliminated with the paternal autosomes. Intrafamilial comparisons between the phenotypic and genotypic estimates provide an assessment of the two causes. Mitotic instability causes mosaicism, but does not bias either the $t_{\mathrm{g}}$ or $t_{\mathrm{p}}$ estimates, that is, a family producing few males through PSR action (low $t_{\mathrm{p}}$ ) may transmit the deletion chromosome to $100 \%$ of those males (high $t_{\mathrm{g}}$ ). Inefficient protection of deletion chromosomes from their own action should be evident as a consistent deficit of $t_{\mathrm{g}}$ relative to $t_{\mathrm{p}}$, because of the early elimination of the chromosome during development, that is, a family producing few males through PSR action (low $t_{\mathrm{p}}$ ) will only transmit the chromosome to a fraction of those males (low $t_{\mathrm{g}}$ ). Application of the Wilcoxon signed-rank test to these paired data revealed that consistently significant deficits were observed for chromosomes M008, I008, and E306, thus indicating that these chromosomes may be inefficiently protected from their own action. These three chromosomes also exhibit the same marker profiles for a terminal deletion of the long arm.

Measures of paternal transmission rates for nonfunctional chromosomes were also obtained (Table 6). In the original assays, transmission rates were variable (50$100 \%$ ) for different nonfunctional chromosomes. Immediately after its creation, the transmission rate of F599 was low $(58.9 \%)$, but following subsequent passage of this chromosome through several generations (alternating between males and females), this chromosome 
exhibited a high $(99.1 \%)$ transmission rate in the later analysis. The results for F599, and several of the other nonfunctional chromosomes, demonstrate that small nonfunctional deletion chromosomes can achieve high rates of transmission through males (ie, via sperm) but typically have low transmission through females (ie, via eggs).

\section{Discussion}

Use of site-specific markers for examining deletion derivatives of PSR provides a distinct improvement in the ability to detect the presence/absence of regions of the chromosome. Both the PCR and restriction site assays were efficient and reliable for determining the content of PSR deletion chromosomes. In two cases where a close association was known from restriction analysis of cloned DNA, the PCR and restriction markers were in perfect agreement on this set of deletion chromosomes. The resulting map of PSR generated with these singlesite markers is consistent with previous cytological investigations (Nur et al, 1988; Reed, 1993) and localization of repetitive sequences (Beukeboom and Werren, 1993b). The markers developed in this study detect the presence of insertion sites of the retrotransposon NATE on the PSR chromosome (McAllister, 1995). Based on the number of cloned elements and the number of left and right insertion sites detected by Southern hybridization, at least nine copies of NATE are dispersed throughout the PSR chromosome. Multiple copies of NATE are apparently located in both arms of the submetacentric PSR chromosome, because two sets of markers were deleted independently from the chromosomes. The two independent sets had a disproportionate number of markers; therefore, the smaller set was assumed to be on the short arm and the larger set on the long arm.

The organization of cloned regions provides a basis for comparing the single-site markers developed here to previous data on the distribution of repetitive sequences on the deletion chromosomes. In a cloned region of PSR, the P18 marker is located adjacent to the tandemly repeated sequence PSR79. The deletion profile of this single-site marker indicates that PSR79 is localized to the short arm of PSR, which is consistent with the interpretation of Beukeboom and Werren (1993b). Contents of the other cloned regions of PSR provide further comparisons for two other repeat families. The PSR22 repeat is adjacent to the P41 marker, and the PSR2 repeat is adjacent to the P5 marker. Deletion of these two singlesite PCR markers was not consistent with adjacent repetitive sequences. In several instances, either or both of these markers were deleted and their adjacent repetitive sequences were present. This result suggests that the PSR2 and PSR22 tandem repeats are present in multiple arrays (more than represented in Figure 2). This finding is not entirely surprising, because junctions have been detected between the PSR2 repeat and the PSR18 repeat, which is associated with the centromeric region of the chromosome (Beukeboom and Werren, 1993b; Reed et al, 1994).

The presence/absence of specific markers on the deletion chromosomes provides a basis for localizing regions of the chromosome involved in different biological aspects exhibited by PSR. Two different roles of PSR were examined: ability to cause paternal genome loss and stability of the chromosomes in male wasps. The ability to determine the presence/absence of specific regions relative to the phenotypes of the deletion chromosomes provides a means to localize the underlying genetic regions of PSR function.

The primary difference in the marker profiles of functional and nonfunctional chromosomes was the number of chromosomal regions deleted. Apparently, all nonfunctional chromosomes had deletions in both chromosomal arms. Except for E306, none of the functional chromosomes had detectable deletions in both arms. This observation may be consistent with the 'sink model' of PSR function. Under this model, it is expected that an expanded region or multiple regions of PSR operate in the capacity to bind a product that is needed by the paternal autosomes (Werren, 1991; Beukeboom and Werren, 1993b). Although the general difference between functional and nonfunctionals suggests that the function may be related to overall chromosomal size, the E133 nonfunctional chromosome is clearly inconsistent with this explanation. This chromosome retained the entire long arm, except for having the 10/105 repeats deleted, and was much larger than many of the functional chromosomes. Another problem with the size explanation is that some of the functional chromosomes had as much or more of the long arm deleted than the nonfunctionals. Therefore, a decrease in overall size does not seem responsible for loss of function.

Another difference between functional and nonfunctional chromosomes is the frequency of deletions in the short arm of PSR. All nine nonfunctional chromosomes exhibited deletions in the short arm. However, only four of the 13 functional chromosomes had deletions in the short arm. This finding is suggestive of a region in the short arm of the chromosome being involved in function, because it is always deleted when a chromosome is rendered nonfunctional. However, comparison of marker profiles between functional and nonfunctional chromosomes with short-arm deletions is inconsistent with functionality being determined solely by a single locus in this region. The deletion in the functional chromosome E146 is apparently located nearer the centromere than the deletions in five of the nonfunctional chromosomes.

Based on the profiles of the deletion chromosomes assayed in this study, the region necessary for paternal genome loss appears redundant on the two chromosomal arms. The data suggest that the presence of the distal regions of either the short arm (around repeat NL5.1) or the long arm (around repeat PSR 10/105) is sufficient for PSR function. This is implied by the observation that all nonfunctional chromosomes have evidence of terminal deletions of both arms, including nonfunctional chromosome E133, which apparently only has terminal deletions of the distal portion of both arms. Furthermore, function is retained by four chromosomes with terminal deletions of the short arm and an intact long arm, eight chromosomes with terminal deletions of the long and an intact short arm, one chromosome with an interstitial deletion leaving both ends present, and one chromosome with P18 missing from the short arm and a substantial deletion of the long arm. The single-site markers greatly improved the characterization of the deletion chromosomes, and should assist continued 
efforts to identify the loci controlling paternal genome loss.

In addition to causing paternal genome loss, PSR also has to undergo basic chromosomal functions during mitotic cell divisions. In a study of the progeny from 379 males, the wild-type PSR chromosome was transmitted to an average of $93.5 \%$ of fertilized eggs (Beukeboom and Werren, 1993a). This transmission rate by male wasps is a measure of mitotic stability of the chromosome during development, because mitotic instability causes mosaicism that reduces transmission (Beukeboom et al, 1992). One common phenotype associated with deletion chromosomes is a decrease in transmission (Beukeboom et al, 1992). Variable transmission rates were observed for the functional deletion chromosomes assayed in this study. Differences are correlated with deletions in the two chromosomal arms. Short-arm deletion chromosomes had high transmission rates, whereas the rates for longarm deletions were low. Transmission rate of a functional chromosome, therefore, appears to be dependent on the region that is deleted.

A reduced transmission rate of a functional deletion chromosome may result from an inability to protect against PSR action (paternal genome loss) during the first chromosomal division of a developing zygote. Estimates of functional deletion chromosome transmission rates were obtained by two methods, a phenotypic and genotypic assay. Both assays estimate the number of fertilized eggs that were converted to male progeny due to the action of PSR. The phenotypic estimate is based on the sex ratio of these progeny, and assumes that all fertilized eggs that were converted to males also retained the chromosome. However, the genotypic estimate is based on the actual frequency of male progeny that carry the chromosome. One potential cause of a reduction in the genotypic relative to the phenotypic estimate is inefficient protection of the chromosome against its own action. In other words, the deletion chromosome causes loss of the entire paternal genome, including itself. All of the functional deletion chromosomes exhibited about the same overall reduction in the genotypic transmission rate relative to the phenotypic rate. However, three chromosomes (M008, I001, and E306) exhibited significant pairwise reductions in genotypic relative to phenotypic transmission in each family. All three of these chromosomes also exhibit terminal deletions in the long arm of PSR. The E242 chromosome, which apparently has an internal deletion of the long arm, did not have a significant pairwise reduction in the genotypic transmission rate. From these results, it appears that a region of the long arm may play a role in protecting against loss in the developing zygote.

Comparison of transmission rates between functional and nonfunctional deletion chromosomes is also suggestive of long-arm deletions causing a unique phenotype for chromosomes that retain the ability to cause paternal genome loss. High transmission rates were observed for several nonfunctional chromosomes with apparently large deletions of the long arm. Transmission of the F599 chromosome was examined most extensively, and its transmission rate increased over several generations of maintenance. Although the F599 chromosome has deletions in both chromosomal arms, it is stable during mitotic cell divisions as represented by its presence in nearly $100 \%$ of fertilized eggs. On the other hand, an intensive selection regime lasting 10 generations failed to increase transmission of two functional chromosomes (E306 and E242) with long-arm deletions (McAllister, unpublished data). This further suggests that a region within the long arm may be necessary to protect functional chromosomes from their own action, whereas this region would not affect transmission of nonfunctional chromosomes.

Although this analysis did not clearly resolve the genetic basis of PSR's action, these markers provide insight into regions of the chromosome that perform different biological roles. One very interesting finding was the apparent number and dispersion of regions that govern different phenotypic effects of the chromosome. It appears that multiple regions may be responsible for paternal genome loss, and that a region in the long arm may be involved in protection against loss in the zygote. These findings indicate that complex interactions among regions of this chromosome are involved in maintaining PSR's ability to drive. However, further studies are needed to reveal the genetic mechanisms involved.

\section{Acknowledgements}

We appreciate the provision of a deletion strain by M-J Perrot-Minnot, and the technical assistance of J Fox. This work is supported by grants from the US National Science Foundation to BFM and JHW.

\section{References}

Beukeboom LW (1994). Phenotypic fitness effects of the selfish B chromosome, paternal sex ratio (PSR) in the parasitic wasp Nasonia vitripennis. Evol Ecol 8: 1-24.

Beukeboom LW, Werren JH (1993a). Transmission and expression of the parasitic paternal sex ratio (PSR) chromosome. Heredity 70: 437-443.

Beukeboom LW, Werren JH (1993b). Deletion analysis of the selfish B chromosome, paternal sex ratio (psr), in the parasitic wasp Nasonia vitripennis. Genetics 133: 637-648.

Beukeboom LW, Reed KM, Werren JH (1992). Effects of deletions on mitotic stability of the paternal-sex-ratio (PSR) chromosome from Nasonia. Chromosoma 102: 20-26.

Camacho JP, Sharbel TF, Beukeboom LW (2000). B-chromosome evolution. Philos Trans $R$ Soc Lond Ser B 355: 163-178.

Eickbush DG, Eickbush TH, Werren JH (1992). Molecular characterization of repetitive DNA sequences from a $\mathrm{B}$ chromosome. Chromosoma 101: 575-583.

Hastings IM (1994). Selfish DNA as a method of pest control. Philos Trans R Soc Lond Ser B 344: 313-324.

Hurst GD, Werren JH (2001). The role of selfish genetic elements in eukaryotic evolution. Nat Rev Genet 2: 597-606.

Jaenike J (1996). Sex-ratio meiotic drive in the Drosophila quinaria group. Am Nat 148: 237-254.

Jones RN (1991). B-chromosome drive. Am Nat 137: 430-442.

Jones RN, Rees H (1982). B chromosomes. Academic Press: New York.

Lyttle TW (1977). Experimental population genetics of meiotic drive systems. I. Pseudo-Y chromosomal drive as a means of eliminating cage populations of Drosophila melanogaster. Genetics 86: 413-445.

Lyttle TW (1991). Segregation distorters. Annu Rev Genet 25: 511-557.

Lyttle TW (1993). Cheaters sometimes prosper: distortion of Mendelian segregation by meiotic drive. Trends Genet 9: 205-210. 
McAllister BF (1995). Isolation and characterization of a retroelement from a B chromosome (PSR) in the parasitic wasp Nasonia vitripennis. Insect Mol Biol 4: 253-262.

Nur U, Werren JH, Eickbush DG, Burke WD, Eickbush TH (1988). A 'selfish' B chromosome that enhances its transmission by eliminating the paternal genome. Science 240: 512-514.

Perrot-Minnot M-J, Werren JH (2001). Meiotic and mitotic instability of two EMS-produced centric fragments in the haplodiploid wasp Nasonia vitripennis. Heredity 87: 8-16.

Reed KM (1993). Cytogenetic analysis of the paternal sex ratio chromosome of Nasonia vitripennis. Genome 36: 157-161.

Reed KM, Beukeboom LW, Eickbush DG, Werren JH (1994). Junctions between repetitive DNAs on the PSR chromosome of Nasonia vitripennis: association of palindromes with recombination. J Mol Evol 38: 352-362.

Reed KM, Werren JH (1995). Induction of paternal genome loss by the paternal-sex-ratio chromosome and cytoplasmic incompatibility bacteria (Wolbachia): a comparative study of early embryonic events. Mol Rep Dev 40: 408-418.

Silver LM (1985). Mouse $t$ haplotypes. Annu Rev Genet 19: 179-208.
Silver LM (1993). The peculiar journey of a selfish chromosome: mouse $t$ haplotypes and meiotic drive. Trends Genet 9: 250-254.

Werren JH (1991). The paternal-sex-ratio chromosome of Nasonia. Am Nat 137: 392-402.

Werren JH, van den Assem J (1986). Experimental analysis of a paternally inherited extrachromosomal factor. Genetics 114 2170-2233.

Werren JH, Nur U, Eickbush DG (1987). An extrachromosomal factor causing loss of paternal chromosomes. Nature 327: 75-76.

Werren JH, Nur U, Wu C-I (1988). Selfish genetic elements. Trends Ecol Evol 3: 297-302.

Werren JH, Skinner SW, Charnov EL (1981). Paternal inheritance of a daughterless sex ratio factor. Nature 293: 467-468.

Werren JH, Stouthamer R (2002). PSR (paternal sex ratio) chromosomes: the ultimate selfish genetic elements. Genetica 117: 85-101.

Wu C-I, Hammer MF (1991). Molecular evolution of ultraselfish genes of meiotic dive systems. In: Selander RK, Clark AG, Whittam TS (eds) Evolution at the Molecular Level, Sinauer Associates: Sunderland, MA. pp 177-203. 